pations, we may claim for the new treatment that it is a substantialadvance upon older methods, and that its results, though not seldom disappointing, are sometimes brilliant beyond expectation. Those results will be far more solid and enduring when the disease is more often detected in its earlier stages, and when treatment is entered upon while the local damage is still slight and constitutional involvement absent or trivial. In this disease time is a factor of the first magnitude. The earlier manifestations of tubercle are comparatively amenable to treatment, the later are too often irremediable.

One of the most hopeful features of present day medical science is the ever-increasing closeness of association between clinical medicine and pathology. Never before did the hos. pital and the laboratory work so fully hand in hand, never before were observation and experiment so fruitfully combined. Clinical physicians are grateful for the large amount of assistance which pathology gives them in the post-mortem room in interpreting the problems of disease, nor are they insensible of the somewhat more limited assistance which pathology gives them at the bedside. The physician, without the aid of the pathologist, may practise-perhaps successfully practise-an art : he can never expound a science. The pathologist, without the aid of the physician, often finds the facts of the post-mortem room a meaningless tangle, or at best a riddle of which the solution can only be guessed. Patho!ogy is an invaluable aid to clinical medicine, but its function is in the main critical, explanatory, and corrective, rather than dominant or regulative. "The art of medicine," said Sydenham, "is to be properly learnt only from its practice and its exercise." In our profession experience is the supreme test, the bedside is the most important training ground; tlie course of disease and how it can be modified by treatment are in many cases ultimate facts, which no theory can shake and no argumentation can alter.

To seek to anticipate the progress which awaits our art in the coming years, to endeavour to cast the medical horoscope of the twentieth century would be a tempting task, but I can only lightly touch it to day. That the outlook is hopeful may be safely inferred from the fact that medical science is to-day in possession of new and fruitful ideas and of methods which are still unexhausted. The serum treatment of disease and the doctrine of internal secretion are still only in their infancy. The prevention of tuberculosis, which is really only a phase in the application to medicine of aseptic principles already dominant in surgery, is within sight, although the goal is still distant. The brilliant illumination which Golgi and his school have shed upon the structure and functions of the nervous system may point the way to some therapeutic progress in this mos! difficult and baffling field. We may well contemplate the future with confidence, feeling assured that whatever disappointments the twentieth century may have in store for us, it can hardly fail to witness the growth and development of our art, and a corresponding gain to the race in comfort, happiness, and longevity.

While I would urge you to spare no labour in the endeavour to master every branch of your art, I think I might invoke the authority of my predecessor in this chair in cautioning you against a narrow professionalism, and in recommending as wide an outlook as possib'e upon the ever-broadening field of knowledge. 'The late Dr. Cuming owed no small part of his unique influence to his general attainments and breadth of culture. I know that the medical student of to day is a muchlectured and much-examined individual. It may secm a refinement of cruelty to suggest that he should extend his studies beyond the somewhat capacious limits of the medical curriculum. But onerous as without doubt are his taske, I think he has still a small margin of leisure. Let me plead with you to give some portion of your limited leisure to the study of good literature. When football and cricket, the bicycle and the cue have had proper and adequate attention paid to them, reserve come margin of time for the poets, the essayists, the historians, and the biographers. If you can enjoy Homer and Horace, Molière and Heine, so much the happier are you, but if not, the field of English literature is wide, it abounds in every variety of literary product, and is practically inexhaustible. Make a daily companion of Shakespeare or Wordsworth, or Tennyson, or, if you prefer, of Scott or Emerson or Carlyle-it matters little which you choose, so long as you choose among the best. While leaming the attachments of the latissimus dorsi or the characteristics of electrotonus, do not forget the tale of Troy divine, or the immortal knight of La Mancha or the Prince of Denmark. The great masters of literature will help you to that knowledge of life which is hardly less important to you than a knowledge of your profession. "Most men," says Lowell, " make the voyage of Jife as if they carried sealed orders which they were not to open until they were fairly in mid ocean." Literature is the record of the accumnlated experience of mankind rendered immortal by the ealt of genius. Its study can hardly fail to make you more flexible in mind, more tolerant in opinion, more sympathetje in heart. It will also help you to a juster estimate of yonr profession and of your own place in human society. Yonr business will be first and chiefly to prevent disease, to cure disease, and to relieve suffering, but you may also each in your sphere do something to hold up the standard of liberal ealture and to pass on the torch of knowledge to those who will come after us.

\section{SOME REMARKS ON RINGWORM.}

WITH ESPECIAL REFERENCE TO THE EARLY STA(iE OF ATTACK OF 'THE HAIR AND THE PRODUCTION OF PUSTULAR

$$
\text { INFLAMMATION.* }
$$

T. COLCOTT FOX, and FRANK R. BLAXALL,

M.B.Lond., F.R.C.P.,

Physician for Diseases of the Skin to M.D.Lond, D.P.H.Camb., the Westminster Hospital and to the Westminster Hospital the Skin Department of the

Paddington Green Children's Hospital.

THE following remarks fall under two heads. In the first place we desire to demonstrate some drawings which portray the various stages of the attack of the hair by the difierent fungi ; and, secondly, we offer some new observations on the asscciation of endothrix fungi with kerion.

Preparation of specimens.-By way of preface we may add a word on the preparation of specimens. Stained specimens are striking as microscopical objects, and are invaluable for preservation, but their preparation often demands close attention and time. The fungus located in the interior of the bair sometimes stains with difficulty, and in removing the excess of stain such fungus is very apt to be decolorised. The examination of infected hairs in liquor potass $x$ (B.P.) is easy and convenient, especially, for instance, when it is desjrable to study a griat number of hairs from a given case. It is also extremely satisfactory as a temporary means of displaying the fungus and its location. The fungus resists the disintegrating action of the potash, and the epithelial structures may be completely dissolved away by prolonged soaking, leaving the fungus intact, and the latter may then be stained.

Exhibition of Drauings. - These drawings are more or less diagrammatic, but they have been carefully made from stained specimens. They show very clearly the fundamental similarity in the attack of the fungi in all cases, whilst. illustrating the minor variations characteristic of each gronp.

\section{Microsporon Audouin I.}

If from a very early macule on the scalp the apparent]y healthy hairs are carefully epilated, a proportion of them will afford material for the stud $\bar{y}$ of the earliest stages of the attack. Similar features will be met with time after time. Fig. I is drawn from a very happy specimen, and shows a quantity of plain branching mycelium ramifying in the quathelium at the mouth of the follicle and encircling the hair throughout its intrafollicular portion. The hair itself is not yet attacked. Fig. 2 illustrates specimens which can be yet attacked. profusion. The early follicular mycelium has disappeared for the most part, just as it disappears from the surface epithelium, but the intrafollicular portion of the hair is leing attacked. The precise site and method of attack have been the subjects of much dispute. We are not disposed to dogmatise beyond pointing out that in mally hundreds of specimens we have nnlv nheprved in micsosporon * Read at the meeting or the British Medical Association at Edin bergh (Section of Dermatology), $189^{\circ}$. 
he method here portrayed. The fungus first enters beneath the cuticle in the lower third of the follicle above the bulb, and similarly enters and strips off the cuticle from below apwards towards the mouth of the follicle. It is a remarkable act that when the invading mycelium comes into this

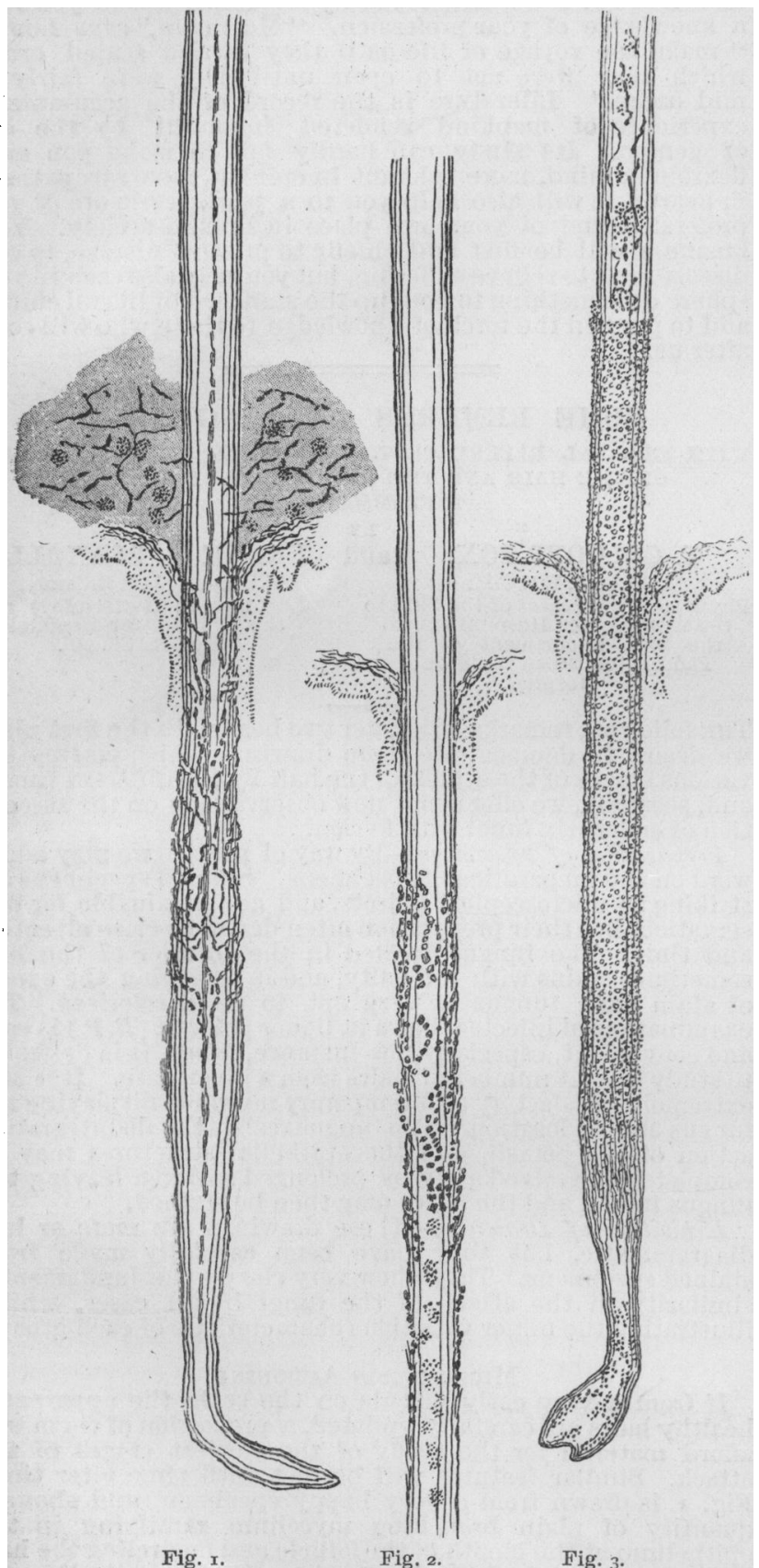

intimate contact with the hair it assumes peculiar characters. It enters beneath the cuticle, which it strips off, and divides up into short segments, sometimes arranged in linear series, but for the most part forming facetted groups. These segments a re at first of large siz with thin walls, and constitute the "giant" or "ghost-like" elements described by Adamson and onrselves. Gradually the segments, as they subdivide, become smaller and smaller, and the groups form, coalesce, and crodinally constitute the characteristic mosaic closely enidiving the hair. At the same time some threads enter and grow between the hair fibres, but this never occurs to the same extent as in endothrix and ectothrix.

The correctness of our description of the method of infection is in dispute and awaits confirmation. You will remember that Sabouraud gives an entirely different account of the matter. He described the mycelia as entering the hair, throwing off lateral branches, which in turn give rise to more delicate processes piercing the cuticle, and then terminating on the outside of the hair in an ectospore, as distinguished from a mycelial endospore. He, therefore, maintains that the microsporon is the only one of the ringworm parasites which is capable of passing through a complete developmental cycle (with the production of ectospores) whilst parasitic on the human subject. If such were the case the microsporon would be clearly and widely separated from the other ringworm fungi.

Fig. 3 portrays the completely infected hair. It will be noticed that the mycelium within the hair terminates in a fringe above the bulb, though the amount of this mycelium varies a great deal in different hairs. In decolorising stained specimens this fringe is apt to be lost. It is characteristic of these hairs to see columns of "spores" arranged on the surface of the root, and occasionally the mycelium within grows right down into the bulb. Our explanation is that the mycelium only grows down in this manner when the hair is dead-that is, separated from the papilla.

The Mycelium. - In microsporon, whether in the macules of glabruus skin, or the scalp, or in the hair follicle, the mycelium is as a rule segmented only at long intervals, or appears quite plain, though it often branches irregularly in a characteristic way. But in, or on, the hair there are numerous exceptions to this rule, and the mycelium is often seen to be segmented at short intervals, forming chains of right-angled or rounded or oval segments. These can generally be distinguished by the expert from the chains of the other kinds of ringworm fungi, but sometimes the resemblance is very close. We exhibited a photograph of a remarkable specimen at the International Congress of Dermatology, $1896 .^{1}$ Sabourand very naturally hesitated to accept this specimen as an example of microsporon, and he refers to the point in the fourth paragraph on page 513 of the Transactions of the Third Congress of Dermatology, 1896. We were, however, able to place the matter beyond dispute by the demonstration of the cultures.

The Fructification. - We have insisted on the desirability of studying the fructifications in the cultures by means of Klat sch specimens, and not by the hanging.drop method. By this means we demonstrated that the fructifications of microsporon, of endothrix, and of ectothrixes, were essentially on the same plan and that the microsporon fructification was not radically different, as maintained by Sabouraud. It was a satisfaction to find our observations in respect to microsporon confirmed by Bodin.

\section{ENDOTHRIX.}

Fig. 4 is drawn from a hair, apparently healthy to the naked eye, extracted from a very early macule on the scalp. It will he seen that the picture corresponds closely with that in Fig. I. The hair in its intrafollicular part is encircled by mycelium somewhat larger than that of microsporon, and with a marked tendency to chain formation. The latter feature is frequently seen in the lesions of tinea circinata.due to these endothrix fungi, and, when met with, is very suggestive. Fig. 6 , for instance, is the drawing of fungus taken from the roof of a vesico-pustule of a tinea circinata of the back of the hand of a woman (Mrs. N.). The culture proved it to be an endothrix. In Fig. I you will also notice the fungus entering beneath the cuticle a little way above the bulb, exactly as in microsporon. Sometimes indications are met with at this early stage of the formation of giant segments, but it is in no way comparable to that so frequently. seen in microsporon, and here there is no division into groups with subsequent confluence to form a mosaic sheath around the hair. The fungus, on the other hand, always assumes the chain formation, and on entering the hair branches dichotomously both towards the bulb and also towards the free end.

We do not assert that this site of attack of the hair is the invariable one, but we have seen it in many specimens, and it is obviously on all fours with that of microsporon. The fungus eventually.packs the hair from end to end (Fig. 5), and 
consequently the hair readily breaks, and characteristic very short stumps are the rule; indeed, they are often broken off short in the mouth of the follicle.

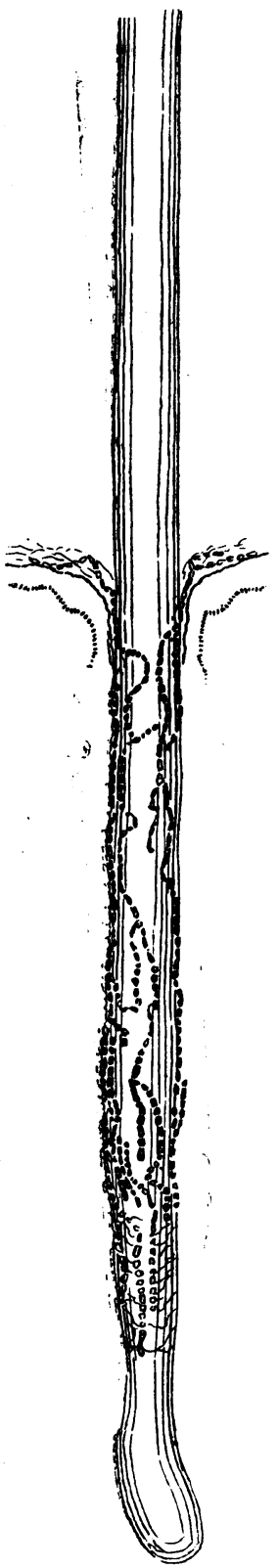

Fig. 4 .

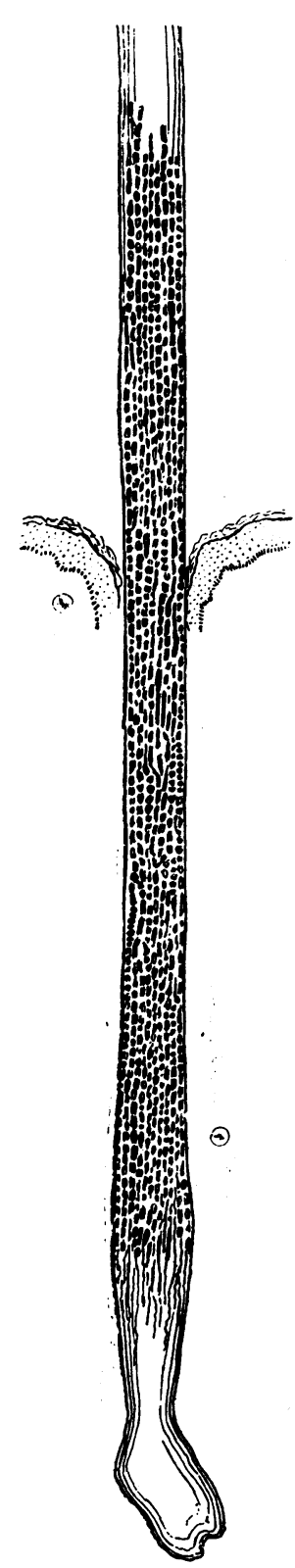

Fig. 5 .
All trace of mycelium exterior to the hair tends to disappear, or is only found exceptionally in the fully infected hairs. Hence the justification of the term "endothrix." In speeimens in which some of this extrapilar mycelium persists the microscopical differentiation of the fungus from some forms of ectothrix is undoubtedly difficult, unless many opecimens are examined, as has been our invariable custom.

It is a notable fact that the cuticle is not, as in microsporon, stripped off, and this makes the staining and preparation of good specimens the more difficult.

The endothrix fungi, as microsporon, meet with resistance to entrance into the succulent live bulb, and in Fig. 5 the mycelium is seen terminating in a fringe above the bulb exactly, as in microsporon. We have constantly met with this appearance. These terminal threads ar feelers are often quite plain and smaller than the rest, and bulbous at the ends as in microsporon. The bulb of the hair may, however, be invaded, but probably only in dead hairs.

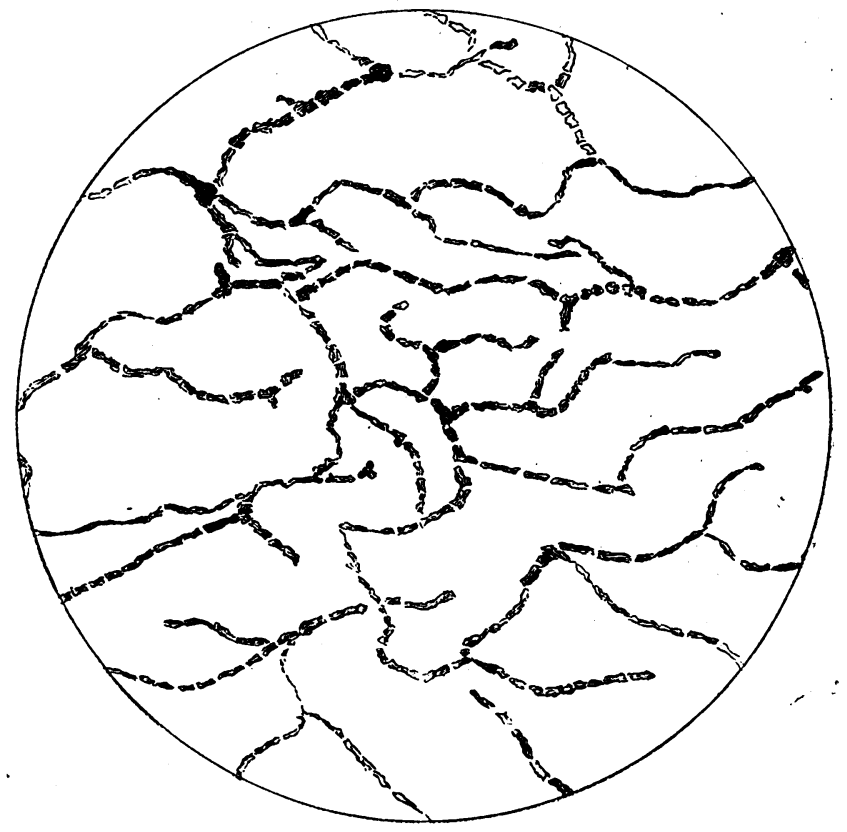

Fig. 6.

We have brought to the meeting a collection of about twenty endothrix cultures, which display in a striking manner the prevailing crateriform type, and illustrate the minor differences of configuration met with. To emphasise the distinct nature of these endothrix fungi it is well to state that all these cases without exception were correctly diagnosed clinically or microscopically before the cultures confirmed the diagnosis.

\section{ECTOThRix}

In examining hairs from any given case, or from;different cases, of microsporon one sees the same picture over and over again, and so with the endothrix tricophytons. On the other hand there is a great difficulty in presenting any one drawing of ectothrix which will convey a complete picture of the latter fungi. This arises from the fact that much diversity of size and appearance is met with owing to many different fungi being comprised in this group. Moreover, the ectothrix fungi are not common in London, and some are very rare. Specimens from ringworm of the beard, mostly of horse origin, are most frequently seen, and several authors have published illustrations. Fig. 7 is taken from an early stage of kerion of a child's scalp. The dense feltwork of mycelium encircling the intrafollicular portion of the hair is represented in the upper portion of the figure $(a)$, as brought away by epilation. In the lower portion (b) the fungus is seen entering beneath the cuticle exactly as in microsporon and endothrix. The fungus is somewhat larger than in microsporon, bat otherwise the picture is very similar. All the specimens obtained from this case were in this comparatively early stage of attack, but in a photograph from another case published in the British Journal of Dermatology, vol. 8 (plate v, fig. I) the fungus is seen to have formed a very dense circumpilar sheath in which the chain formation which existed was masked by the aggregation of the segments. The chain formation, however, was evident within the hair. Figure 8 portrays such appearances. Here the chain formation so prominent in ectothrix and endothrix is clearly seen, but the persistence of the circumpilar sheath of fungus warrants the application of the term ectothrix in distinction to endothrix. The degree to which the interior of the hair is implicated varies:in different cases. Figure g displays a hair taken from a case of ringworm of the horse $g$ and demonstrates the large sized chain formation on and in the hait. : : $: 1 ! \ldots$ 
A point has been made concerning the differences in the mo le and point of attack in microsporon on the one band and endothrix and ectothrix on the other. Whilst repeating that we do not wish to dogmatise on this somewhat difficult matter, we have ample evidence that in all the mode and site of attack may be similar, that is, entering beneath the cuticle o the hair in the lower third of the intrafollicular portion just above the bulb.

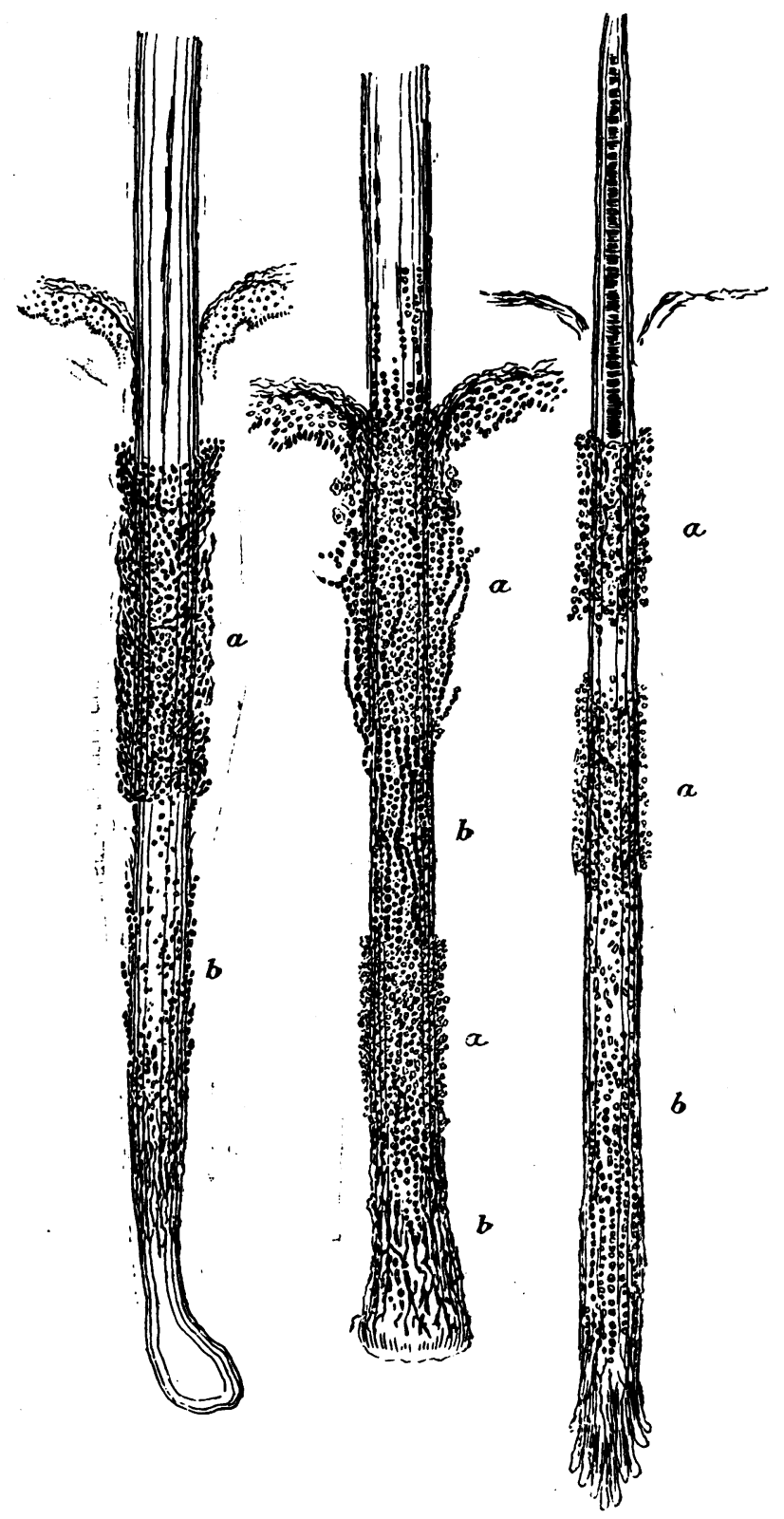

Fig. 7.

Fig. 8.

Fig. 9.

With regard to the presence of a mycelial fringe within the hair above the bulb in the ectothrix infected hair, it is uncommon in our experience, but we have seen it several times. The explanation of this rarity appears to be that these fungi cause so much inflammation as a rule that the bulb is rapidly detached and the fungus rapidly grows down. It is quite possible that the detachment occurs so early that the fungus may sometimes enter the hair by the root.
New ObigRvations on the Association OF the Endothrix FUNGI with Kerion.

We will conclude our remarks with the record of some new observations on the association of the endothrix fungi with kerion. We have met with five cases of spontaneous typical kerion in association with endothrix fungi, and one of us demonstrated a case at the Dermatological Society of London, ${ }^{2}$ so that there should be no doubt as to the clinical facts. After the examination of many hairs in each case we found the fungus in appearance and mode of attack microscopically indistinguishable from endothrix, and the cultivations have confirmed this view and given us well-marked crateriform cultures. Clinically in four of the cases we could only say that we had to do with kerion, as there was nothing distinctive about the configuration of the parent and satellite patches. In ore case, however, long under observation and not quite so highly inflamed as the others, we observed in addition to the main patches pustules perforated by diseased hairs dotted about the scalp singly and in little groups in a manner very suggestive of endothrix.

The causes of this excessive inflammation and pus formation in ringworm remain to be worked out. Sabouraud insisted on the pyogenic properties of certain ectothrix fungi of animal origin, but he combated the idea of kerion being produced by other kinds of fungi. We have certainly cultivated the microsporon from highly inflamed pustular patches of ringworm, which seemed to be rightly designated as kerion. In studying this subject we must recognise that it is not very uncommon to find microsporon patches considerably inflamed secondarily by rubbing and scratching and the application of remedies in susceptible children, and that the presence of pediculi may lead to a secondary pus inoculation, and thus true kerion may be simulated. By kerion, however, we understand the apparently \&pontaneous production of areas of deep-seated conglomerate folliculitis, often studded with pustules and bathed in pus, or covered with crusts. This condition may be due, presumably, to the direct influence of the ringworm fungus, or to the presence of accompanying pyogenic organisms, and it is not always an easy problem to determine, especially in the presence of pediculi, what is the precise agency at work. In the endothrix cases now under prensideration the causation by pediculi was eliminated, and the kerion must have been due either to the fungus or other

pyogenic organisms. may be associated with the presence of the endothrix fung we may mention two cases of vesico pustular tinea circinata due to these fungi. Dr. Fremlin kindly sent us a woman and child with marked vesico-pustular tinea circinata, from which we cultivated an endothrix fungus. ${ }^{3}$ We have lately met with a similar case on a woman's hand (Mrs. N.), and again cultivated the endothrix fungus.

NoTE.-Since this paper was written we have seen a baby, aged 6 weeks, with a huge patch of tinea circinata with a vesicular margin covering the greater part of the vertex of the scalp due to an endothrix fungus. $\mathrm{It}$ is interesting to note scalp due to an endothrix fungus. had never been out of the house or mixed with other children. No clue whatever to its origin from animals, or otherwise, could be made out. NOTES AND REFERENCES.

I Depicted Plate II NOTES AND REFERENCES. the British Journal of ' Depicted Plate II, figure 4, of our paper in the British Journ, July Dermatology, Nos. 93, 96, Dermatology, February, 1898, Case 2).

A VAccination Victory_-Our contemporary, the Philadelphia Medical Journal, publishes the following letter to its editor from the Secretary to the Superior Board of Health of Porto Rico: "San Juan, P.R., October 23rd, 1899. - Sir, I have the honour to inform you that the existence of a single case of small-pox is at this moment unknown on this island. Nine months ago a serious epidemic was threatened and the disease prevailed over the whole island. Since then 800,000 vaccinaiions have been performed. It is possible to stamp out smallpox in Spanish-American countries. There has been no case of yellow fever all summer. No serious diseases have followed the hurricane of Angust .8th.-Very respectfully, Geo. G. Groff, Secretary." Our contemporary speaks of this triumph over small-pox as " a great victory in Porto Rico, and one of which we may be as proud as of winning any battle." 